\title{
Influence of spatial dispersion on surface plasmons, nanoparticles and grating couplers
}

\author{
Armel Pitelet, Emmanuel Centeno, and Antoine Moreav* \\ Institut Pascal, Université Blaise Pascal, \\ 4 avenue Blaise Pascal, \\ 63178 Aubière, France \\ Nikolai Schmitt and Claire Scheid \\ Université Côte d'Azur, Inria, CNRS, LJAD, \\ 2004 Route des Lucioles, \\ 06902 Sophia Antipolis France \\ Dimitrios Loukrezis and Herbert De Gersem \\ Institute for Accelerator Science and Electromagnetic Fields (TEMF), \\ Technische Universität Darmstadt, \\ 64289 Darmstadt, Germany \\ Cristian Ciracì \\ Center for Biomolecular Nanotechnologies, \\ Istituto Italiano di Tecnologia, \\ 73010 Arnesano (LE), Italy
}

\begin{abstract}
Recent experiments have shown that spatial dispersion may have a conspicuous impact on the response of plasmonic structures. This suggests that in some cases the Drude model should be replaced by more advanced descriptions that take spatial dispersion into account, like the hydrodynamic model. Here we show that nonlocality in the metallic response affects surface plasmons propagating at the interface between a metal and a dielectric with high permittivity. As a direct consequence, any nanoparticle with a radius larger than $20 \mathrm{~nm}$ can be expected to be sensitive to spatial dispersion whatever its size. The same behavior is expected for a simple metallic grating allowing the excitation of surface plasmons, just as in Woods famous experiment. Finally, we carefully set up a procedure to measure the signature of spatial dispersion precisely, leading the way for future experiments. Importantly, our work suggests that for any plasmonic structure in a high permittivity dielectric, nonlocality should be taken into account.
\end{abstract}

For more than a century now, Drude's model[1, coupled to Maxwell's equations 2], has been able to describe very accurately the optical response of metals, even for quite extreme geometries [3 7]. Many advanced theories describing metal-vacuum interfaces [8, 9] have been developed during the second half of the twentieth century, especially with the development of Electron Energy Loss Spectroscopy (EELS) which provided experimental data to better ground the theoretical discussions [10 12 . These new approaches were able to take into account complex phenomena, like spatial dispersion or electron spill-out, and allowed to better understand the success of Drude's model. It turns out in fact that the Drude's theory is the zero-th order approximation of all more advanced descriptions introduced later on. All these studies however, seemed to conclude that spatial dispersion and spill-out have a limited impact 13 16 on surface plasmons (SPs) such that optical experiments were not likely to show any difference from Drude's predictions [17 19]. Moreover, because most resonances in metallic structures can actually be explained as cavity resonances of some sort for SPs, it has become widely accepted that plas-

\footnotetext{
* antoine.moreau@uca.fr
}

monic resonances could be very accurately described by Drude's model. For decades, then, there has not been any urge to adopt advanced descriptions of the response of metals in plasmonics. Only in the case of metallic clusters, due to the extremely small size, spatial dispersion and spill-out were expected to play a significant role, requiring the most advanced descriptions $13,20,22$.

However, a recent experiment with film-coupled nanoparticles showed that the frequency of the resonance of modes that are localized in small volumes (of the order of $1 \mathrm{~nm}^{3}$ ) is simply not correctly predicted by Drude's model, whereas the linearized hydrodynamic model [23], in its simplest formulation (the Thomas-Fermi approximation) seemed to be accurate enough. This is the case even with an optical excitation and relatively large metallic particles. This can be linked to the fact that the small gaps between the nanoparticle and the metal support a gap-plasmon - a guided mode which is particularly sensitive to spatial dispersion because it has a very large wavevector 24,28 , whatever the frequency. This allows to better understand why small gaps, which are more and more common in plasmonics [3, 5, 29, 31, may require more advanced descriptions of the metallic response. Furthermore, this explains why the hydrodynamic model, despite its well doc- 
umented deficiencies 32, is probably a good replacement for Drude's model in plasmonics 33, 34: high wavevector plasmonic modes enhance spatial dispersion effects $26-$ 28, but not the impact of the spill-out, especially since noble metals present a high extraction work. The spatial dispersion which arises from the repulsion between free electrons inside the metal is taken into account accurately by a hydrodynamic model, which presents in addition the advantage of being relatively easy to implement numerically 35 38.

Here we show that the impact of spatial dispersion on the SP propagating at the interface between a metal and a dielectric is enhanced when the refractive index of the dielectric is large. We show, as a direct consequence, that even large nanoparticles can be expected to be sensitive to nonlocality and that, using a grating coupler, it is theoretically possible to estimate the main parameter of the linearized hydrodynamic model, in a situation where other more complex phenomena can be ruled out. We underline that such a well controlled setup differs strongly from previous experiments which all involved chemically synthesized nanoparticles 20, 39 41 whose geometry cannot always be fully controlled.

\section{SURFACE PLASMON}

In this first part we study the influence of spatial dispersion on a SP propagating along a metal-dielectric interface (especially for dielectrics with high refractive indices). A SP can be seen as current loops propagating beneath the surface of a metal. Such a phenomenon is accompanied by an electromagnetic field in the metal (with relative permittivity $\varepsilon_{\mathrm{m}}$ ) and in the dielectric (with relative permittivity $\varepsilon_{\mathrm{d}}$ ) which is transversely evanescent in both media due to the fact that its effective index $n_{\mathrm{eff}}=\frac{k_{\mathrm{SP}}}{k_{0}}$ is always larger than the refractive index of the dielectric medium.

First, neglecting losses, the dispersion relation can be written as

$$
n_{\mathrm{eff}}=\sqrt{\frac{\varepsilon_{\mathrm{d}} \varepsilon_{\mathrm{m}}}{\varepsilon_{\mathrm{d}}+\varepsilon_{\mathrm{m}}}},
$$

where we assumed a simple Drude model $\varepsilon_{\mathrm{m}}=1-\frac{\omega_{\mathrm{P}}^{2}}{\omega^{2}}$. In this case, the curve of a SP has a horizontal asymptote at $\omega_{\mathrm{SP}}=\frac{\omega_{\mathrm{P}}}{\sqrt{1+\varepsilon_{\mathrm{d}}}}$. It is however unrealistic to neglect the losses inside the metal because the frequency $\omega_{\text {SP }}$ is usually in a wavelength range, the UV, where the interband transitions make the metal highly lossy. As a consequence, a bend-back can be seen on the dispersion curve of the SP, which thus never reaches very high wavevectors (see local dispersion curves in Fig. 1).

However, when the permittivity of the dielectric increases, the frequency $\omega_{\mathrm{SP}}$ decreases. At the same time, losses due to the interband transitions can be expected to be low enough as to enable a support of high wavevector SPs. Such modes are more likely to be sensitive to spatial dispersion 24.

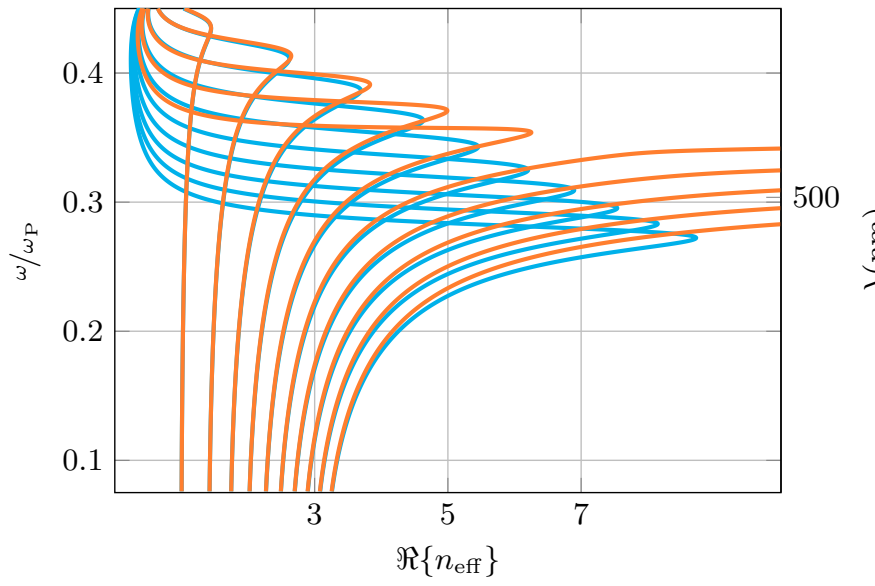

FIG. 1. Surface plasmon dispersion curves (see relation (3p) along a single dielectric - Ag interface, assuming material parameters for silver 42]. The permittivity of the dielectric $\varepsilon_{\mathrm{d}}$ ranges from 1 (most left) to 10 (most right) with a step size 1. The blue lines correspond to the local dispersion $(\Omega=0)$ and the orange ones to nonlocal dispersion $(\Omega \neq 0)$.

In order to take nonlocality into account we rely on the linearized hydrodynamic model for the free electrons, already introduced in previous works [35, 43, 48. The electric current $\mathbf{J}$ inside the metal is linked to the electric field $\mathbf{E}$ by

$$
-\beta^{2} \nabla(\nabla \cdot \mathbf{J})+\ddot{\mathbf{J}}+\gamma \dot{\mathbf{J}}=\varepsilon_{0} \omega_{\mathrm{P}}^{2} \dot{\mathbf{E}},
$$

where $\omega_{\mathrm{P}}$ is the plasma frequency, $\varepsilon_{0}$ the vacuum permittivity, $\gamma$ the damping factor and $\beta$ the nonlocal parameter. The $\beta$ factor represents the increase of the internal pressure in the electron gas due to exchange interaction and Coulomb repulsion. There are actually several theoretical expressions for this parameter 49. We rely on the experimental data available [39, 40, which consistently point to a value of $\beta=1.35 \times 10^{6} \mathrm{~m} / \mathrm{s}$. Finally, the electric current inside the metal can always be considered as an effective polarization $\mathbf{P}_{\mathrm{f}}$ due to the free electrons and is then given by $\dot{\mathbf{P}}_{\mathrm{f}}=\mathbf{J}$. In that framework, the metal can then be described as a nonlocally polarizable medium.

We use accurate material parameters 42 that allow a distinction between the response of the free electrons, that is subject to spatial dispersion, and the response of bound electrons, that can be considered to be purely local [50]. In the following the metal is always assumed to be silver, which is favorable since silver is less lossy than gold. The total metal polarization reads then $\mathbf{P}=\mathbf{P}_{\mathrm{f}}+\mathbf{P}_{\mathrm{b}}$, where $\mathbf{P}_{\mathrm{b}}=\varepsilon_{0} \chi_{\mathrm{b}} \mathbf{E}$ with $\chi_{\mathrm{b}}$ being the local susceptibility associated to the bound electrons. In order do obtain $\chi_{\mathrm{b}}$, we fit the experimental data of silver permittivity with a generalized dispersion model based on Padé series [51] (which is sufficient to perform nonlocal time-domain simulations) and subtract the local Drude contribution $\chi_{\mathrm{f}}$. Throughout this work, we rely on the DIOGENeS 52] Discontinuous Galerkin Time Domain 
(DGTD) suite 37. Finally we consider additional boundary conditions that are both the most natural (vanishing normal component of the polarization current $\mathbf{J}$ at the metal boundary, thus forbidding free electrons to escape the metal) and the most conservative (they reduce the impact of nonlocal effects) 24].

We first study the influence of nonlocality by considering the dispersion relation of a SP propagating along a perfectly plane dielectric-metal interface. Assuming time dependence of the form $e^{-i \omega t}$, solving relation (2) for $\mathbf{J}$, injecting the result in Maxwell's equations and considering both classical and additional boundary conditions, the nonlocal dispersion reads

$$
\frac{k_{\mathrm{m}}}{\varepsilon_{\mathrm{m}}}+\frac{k_{\mathrm{d}}}{\varepsilon_{\mathrm{d}}}-i \Omega=0 .
$$

Here, $k_{j}=\sqrt{\varepsilon_{j} k_{0}^{2}-k_{\mathrm{SP}}^{2}}, j=\mathrm{m}, \mathrm{d}$, are the vertical components of the SP wavevector and $\Omega$ is the parameter including $\beta$ and thus taking nonlocality into account. Presuming $\Omega=0$ allows to retrieve the usual dispersion relation for SPs, the $k_{j}=k_{j}^{\prime}+i k_{j}^{\prime \prime}$ being here essentially imaginary $\left(k_{j}^{\prime \prime}>>k_{j}^{\prime}\right)$. The expression of this parameter is 24]

$$
\Omega=\frac{k_{\mathrm{SP}}^{2}}{\kappa_{\mathrm{l}}}\left(\frac{1}{\varepsilon_{\mathrm{m}}}-\frac{1}{1+\chi_{\mathrm{b}}}\right),
$$

where $\kappa_{1}^{2}=k_{\mathrm{SP}}^{2}+\left(\frac{\omega_{\mathrm{P}}^{2}}{\beta^{2}}\right)\left(\frac{1}{\chi_{\mathrm{f}}}+\frac{1}{1+\chi_{\mathrm{b}}}\right)$ is the vertical component of the wavevector associated with the longitudinal part of the SP appearing only when considering non-locality 24 .

As can be seen from (4), the parameter $\Omega$ is roughly proportional to the square of $k_{\mathrm{SP}}$, which clearly indicates that the higher the wavevector, the higher the impact of nonlocality. Fig. 1 shows local and nonlocal SP dispersion curves for different values of the dielectric permittivity $\varepsilon_{\mathrm{d}}$ ranging from 1 to 10 . We can easily see that, for a fixed frequency, an increasing $\varepsilon_{\mathrm{d}}$ pushes the SP towards higher $n_{\text {eff }}$, and thus causes the SP to be more sensitive to nonlocality. The leftmost curve, obtained using air as dielectric $\left(\varepsilon_{\mathrm{d}}=1\right)$ clearly shows that the effective index $n_{\text {eff }}$ is too small to show any impact of nonlocality. However, after a certain $\varepsilon_{\mathrm{d}}$ value, the characteristic bendback occurring at the SP frequency $\omega_{\text {SP }}$ just disappears when spatial dispersion is taken into account.

This corresponds exactly to a recent theoretical study [25] which shows that an artificial decrease of the metallic losses can induce exactly the same behavior on the dispersion curves of plasmonic guided modes. This occurs when the impact of nonlocality overcomes the influence of the metallic losses.

The impact of the dielectric's permittivity overcomes the one of losses for two reasons. First, as explained above, increasing $\varepsilon_{\mathrm{d}}$ lowers $\omega_{\mathrm{SP}}$ and thus takes the frequency away from the interband transitions. Additionally, a higher dielectric permittivity directly gives to the SP a higher $n_{\text {eff }}$ and thus enhances the influence of spa- tial dispersion, leading to a large impact of nonlocality even well below $\omega_{\mathrm{SP}}$.

We underline that, despite extensive studies on nanoparticles, such a behavior of the SP has seemingly not been reported previously. Straightforwardly, this suggests that nonlocality will have an impact on (i) the resonances of relatively large nanoparticles of noble metals (with a diameter well above $20 \mathrm{~nm}$, as they can be considered as resonant cavities for the surface plasmon) and (ii) SP grating couplers very similar to the canonical experiment of Wood [53] provided the grating is buried in high index dielectrics.

\section{NANOPARTICLES}

The resonance of large metallic nanoparticles can be interpreted as cavity resonances for the surface mode with a resonance condition $[54$ which can be written as

$$
2 \pi R=m \frac{\lambda_{0}}{n_{\mathrm{eff}}}
$$

or simply as $k_{\mathrm{SP}}=\frac{m}{R}$, where $R$ is the radius of the particle. Such a condition is strictly valid only (i) for a cylinder instead of a sphere and (ii) if the curvature of the particle can be neglected, which is almost never the case. However, this condition being roughly valid even for spherical nanoparticles instead of cylinders [54, 55], it allows to understand that if the wavevector of the SP is influenced by nonlocality, the resonance frequencies of a nanoparticle should be influenced as well, irrespective of its size.

For decades now, the community has actually focused on nanoparticles with a diameter much smaller than 20 $\mathrm{nm} 20$, 41, hoping that enhanced nonlocal effects would take place - since spatial dispersion is linked to supplementary pressure terms in the description of the electron gas 49, 56. However, with very small nanoparticles (i) other effects like the spill-out 222, 33, 57, 58. kick in and (ii) the geometry of the nanoparticles is not well controlled [20, 41]. Given the relatively poor agreement between experiments and the prediction of the hydrodynamic model 41, 59, it seems difficult to consider the hydrodynamic model to be sufficient at such small scales. It may even be a little bit early to introduce further improvements of the hydrodynamic model [60] based on these results. The simple analysis above suggests that larger nanoparticles buried in a high permittivity medium could actually be a better setup to test the hydrodynamic model.

In order to further strengthen this analysis, we used Mie theory [54, 55] and adapted the formalism proposed by Ruppin 61] for metallic cylinders, for which formula 5 is the most relevant. We use the most realistic material parameters possible 42 and a supplementary boundary condition which can be written 24 


$$
\mathbf{P}_{\mathrm{f}} \cdot \mathbf{n}=\left(\mathbf{E}-\left(1+\chi_{b}\right) \frac{\beta^{2}}{\omega_{p}^{2}} \nabla(\nabla \cdot \mathbf{E})\right) \cdot \mathbf{n}=0
$$

where $\mathbf{n}$ is the unitary vector normal to the interface. When the field is decomposed on the cylindrical harmonics, the Mie coefficients 61 are

$$
a_{n}=\frac{\sqrt{\epsilon_{d}} J_{n}\left(k_{d} R\right)\left(J_{n}^{\prime}\left(k_{m} R\right)+\alpha_{n}\right)-\sqrt{\epsilon_{m}} J_{n}\left(k_{m} R\right) J_{n}^{\prime}\left(k_{d} R\right)}{\sqrt{\epsilon_{m}} H_{n}^{\prime}\left(k_{d} R\right) J_{n}\left(k_{m} R\right)-\sqrt{\epsilon_{d}} H_{n}\left(k_{d} R\right)\left(J_{n}^{\prime}\left(k_{m} R\right)+\alpha_{n}\right)}
$$

where

$$
\alpha_{n}=\frac{\frac{n^{2}}{k_{m} R^{2}} J_{n}\left(k_{L} R\right) J_{n}\left(k_{m} R\right)}{-k_{L} J^{\prime} n\left(k_{L} R\right)+\left(1+\chi_{b}\right) \frac{\beta^{2}}{\omega_{p}^{2}}\left[k_{L}^{2} J_{n}^{\prime \prime \prime}\left(k_{L} R\right)+\frac{k_{L}^{2}}{R} J_{n}^{\prime \prime}\left(k_{L} R\right)-\frac{k_{L}}{R^{2}} J_{n}^{\prime}\left(k_{L} R\right)+\frac{2 n^{2}}{R^{3}} J_{n}\left(k_{L} R\right)\right]}
$$

with $k_{m}=\sqrt{\epsilon_{m}} k_{0}, k_{d}=\sqrt{\epsilon_{d}} k_{0}$ and

$$
k_{L}=\sqrt{-\frac{\omega_{p}^{2}}{\beta^{2}}\left[\frac{1}{1+\chi_{b}}+\frac{1}{\chi_{f}}\right]}
$$

We then compute the absorption cross-section as

$$
C_{e}=-\frac{2}{k_{d} R} \sum_{n=-\infty}^{\infty} \Re\left(a_{n}\right) .
$$

We underline that when $\alpha_{n}=0$, the expression (7) reduces to the regular Mie expression.

We have computed the local and nonlocal response of nanoparticles with different sizes when they are in water (as is common) or in $\mathrm{TiO}_{2}$ (see Fig. 2). The latter is a good choice to enhance the influence of spatial dispersion because of its high refractive index. Its permittivity $\varepsilon_{\mathrm{TiO}_{2}}$ is described by a generalized dispersion model fitted to experimental data corresponding to thin films of $\mathrm{TiO}_{2}$ grown by atomic layer deposition 62 . This results in a real part of the refractive index of $\mathrm{TiO}_{2}$ comprised between 2.25 and 2.5 over a wavelength spectrum ranging from $2000 \mathrm{~nm}$ to $400 \mathrm{~nm}$. The extinction coefficient is of the order of $10^{-7}$.

In water, nonlocality has a noticeable impact only for a radius approaching $10 \mathrm{~nm}$, whereas in $\mathrm{TiO}_{2}$ nonlocality tends to blueshift all the resonances of more than 4.8 $\mathrm{nm}$ in wavelength even for a radius of $100 \mathrm{~nm}$. We stress here that a high index dielectric is able to sufficiently enhance the magnitude of the nonlocal effects to make it observable on the response of particles/cylinders 5 times larger than the ones usually considered by the community. This should be enough to rule out any other effect like the spill-out and with such a large size, the geometry of the nanoparticles are better controlled - or the nanoparticles could even be probed individually [55, 59.

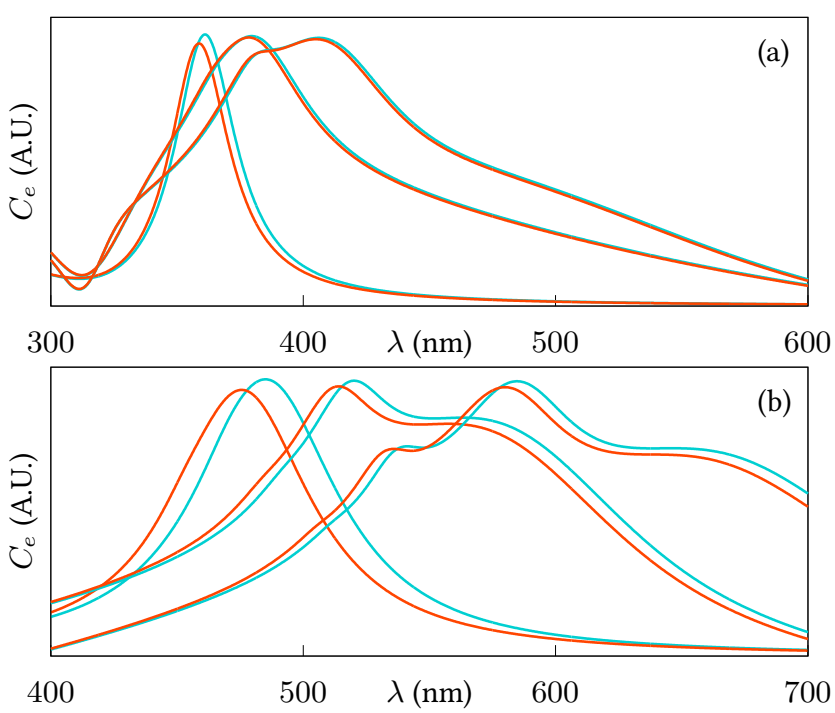

FIG. 2. Local (blue) and nonlocal (orange) absorption for a cylinder cross-section of radius (from left to right) $R=10,50$, $100 \mathrm{~nm}$ as a function of the impinging wavelength $\lambda$ using (a) : water or (b) : $\mathrm{TiO}_{2}$ as dielectric. The theoretical blueshifts (for the maxima) are from left to right 2.1, 1.2, $0.9 \mathrm{~nm}$ on (a) and $9.3,6.0,4.8 \mathrm{~nm}$ on (b).

\section{GRATING COUPLER}

We now discuss the structure shown on Fig. 3(a), which is a simple 1D metallic grating buried in a high index dielectric with infinite extent in the lateral directions. Normal incident illumination is assumed from the top and we recover the zero-th reflected order for a broadband spectrum of the incident wavelength. In such a simple configuration many diffraction orders, including evanescent ones, are excited. 


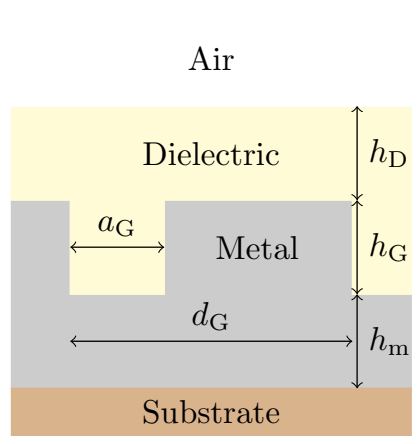

(a)

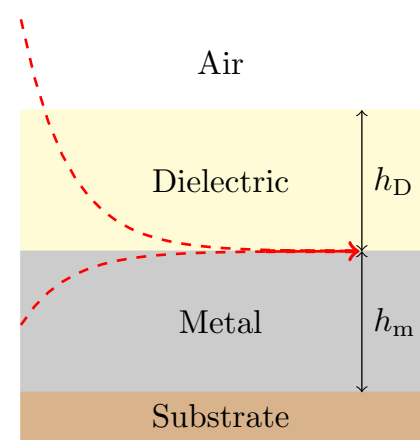

(b)
FIG. 3. (a): Schematic representation of a grating coupler. (b): Schematic representation of a SP propagating along a dielectric - metal interface, taking the finite height of the dielectric into account. The red dashed line illustrates the SP's magnetic profile. While the SP propagates along the dielectric - metal interface, the upper evanescent tail extends into air but the lower one does not extend into the substrate allowing to neglect it in computations.

For the $m$-th order, and since we only consider normal incidence here, the coupling condition to the SP can be simply written as

$$
k_{\mathrm{SP}}=m \frac{\lambda}{d_{\mathrm{G}}},
$$

where $\lambda$ is the wavelength, $m$ the diffraction order and $d_{\mathrm{G}}$ the grating pitch. Such a relation is valid only for a very shallow grating when the surface mode can be considered undisturbed. It is equivalent to assuming that the spatial periodicity of the grating is a multiple of the periodicity of the SP. Such a condition is proper to the periodicity of the structure 63 and thus is expected to be valid whether spatial dispersion (which will modify $k_{\mathrm{SP}}$ ) is taken into account or not. This allows to find the wavelength $\lambda_{c, m}$ for which the grating is able to excite the SP

$$
\lambda_{\mathrm{c}, m}=\frac{\Re\left(n_{\mathrm{eff}}\right)}{m} d_{\mathrm{G}},
$$

where $n_{\text {eff }}=\frac{k_{\mathrm{SP}}}{k_{0}}$ is the SP effective index.

Each time this condition is satisfied, a dip due to the excitation of a SP will appear in the reflectivity. Spatial dispersion should cause blueshifts of the resonances with respect to a fully local approach because the effective index is always smaller when nonlocality is taken into account. We define this blueshift as the positive quantity $\Delta \lambda_{\mathrm{c}, m}=\lambda_{\mathrm{c}, m}^{\text {local }}-\lambda_{\mathrm{c}, m}^{\text {nonlocal }} \propto n_{\mathrm{eff}}^{\text {local }}-n_{\mathrm{eff}}^{\text {nonlocal }}$. As the different resonances correspond to different orders being coupled to the SP, the corresponding wavevectors will be different and thus the impact of spatial dispersion will change from one resonance to another. To better understand the impact of nonlocality on the resonances, it is crucial to be able to identify them, hence the interest in the relation 12 . We have thus taken the finite thickness of the dielectric layer into account by computing the properties of the guided mode of the non-corrugated structure (see Fig. 3(b)) using an open-access numerical tool [36, 64. Aiming for a proper interpretation of the resonances, we have then carefully increased the depth of the grating, in order to allow a thorough physical discussion (see Fig. 5). We use Rigorous Coupled Wave Analysis (RCWA) 65, 66] for these simulations.

\section{A. Parameters of the grating coupler}

\section{Materials}

Although we restrict ourselves to silver in the scope of this work, we underline that gold or any other metal and even semi-conductors 67. (claiming an equivalent electronic mean free path 68) could have been used and would have led to the same conclusions. Again, in order to observe the largest possible effects, we consider $\mathrm{TiO}_{2}$ as a high permittivity dielectric.

\section{Thickness of the dielectric and metallic layer}

We have taken $h_{\mathrm{m}}=150 \mathrm{~nm}$ for the thickness of the metallic layer, thus being several times thicker than the skin depth and ensuring that the substrate does not play any significant role.

The thickness of the dielectric $h_{\mathrm{D}}$ layer is a distinctly more crucial parameter. In order to excite a SP with the highest possible wavevector, a dielectric of the highest possible thickness would be desirable. However, since the dielectric layer is finitely thick, increasing $h_{\mathrm{D}}$ leads to a higher number of classical guided modes. That hinders a clear interpretation of the resonances or renders it even impossible. Luckily, since we are considering the excitation of high wavevector guided modes that show a fairly low vertical extension, a thickness of $h_{\mathrm{D}}=85.0 \mathrm{~nm}$ turns out to be a good trade-off. No other guided mode than the SP exist for this choice of $h_{\mathrm{D}}$ in most of the spectrum.

Fig. 4(a) shows the dispersion curve for a mode propagating at an air- $\mathrm{TiO}_{2}-\mathrm{Ag}$ interface for $h_{\mathrm{D}}=85 \mathrm{~nm}$ with and without spatial dispersion. Obviously, the difference with the dispersion curve of the $\mathrm{SP}$ at a $\mathrm{TiO}_{2}-\mathrm{Ag}$ interface is small. The impact of nonlocality is clearly the same and the bend-back disappears.

\section{Grating pitch}

Using the coupling condition 12 and the local and nonlocal dispersion curves as shown in Fig. 4(a), a raw estimation of the blueshift due to spatial dispersion can be made. Fig. 4(b) shows the expected blueshift for different diffraction orders and sweeps over the grating pe- 


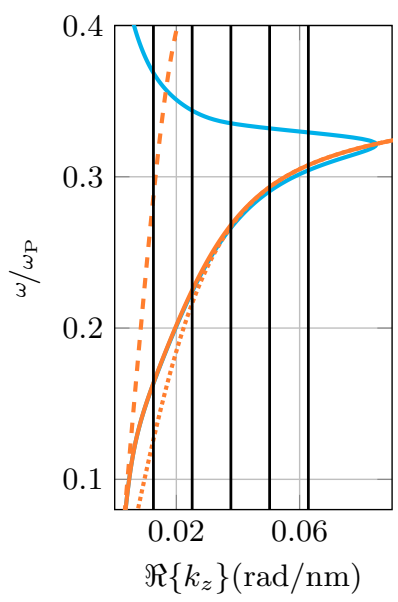

(a)

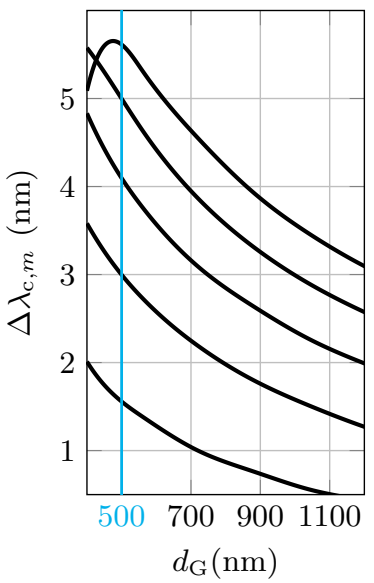

(b)
FIG. 4. (a): Dispersion relation of a SP propagating at an air $-\mathrm{TiO}_{2}-\mathrm{Ag}$ multilayer. Local results are in blue and nonlocal ones are in orange. For comparison, the two dashed lines are the nonlocal dispersion relation respectively for an Air $-\mathrm{Ag}$ interface (left) and a $\mathrm{TiO}_{2}-\mathrm{Ag}$ interface (right). The black vertical lines indicate the coupling condition given by 12 for a grating pitch of $500 \mathrm{~nm}$ considering the five first diffraction orders ( 1 to 5 from left to right). (b): Estimation of the blueshift $\Delta \lambda_{c, m}$ as a function of the grating pitch $d_{\mathrm{G}}$. $m$ varies 1 to 5 (bottom to top).

riodicity $d_{\mathrm{G}}$. The higher the diffraction order, the higher the wavevector of the excited SP - which leads to an increased impact of nonlocality. We have finally chosen a pitch length $d_{\mathrm{G}}=500 \mathrm{~nm}$ according to a maximum of the predicted blueshift of about $5.5 \mathrm{~nm}$ for $m=5$. Keeping the pitch below the shortest working wavelength guarantees that only the zero-th order propagates, even if the evanescent orders of diffraction are coupled to the SP. In other words, all of the non-reflected light must be absorbed by the guided modes along the structure.

\section{Groove width and grating thickness}

The determination of the two remaining geometrical parameters, i.e. the groove $a_{\mathrm{G}}$ and the grating thickness $h_{\mathrm{G}}$, is less straightforward. We have to establish a tradeoff, such that the excitation of the SP for different orders of diffraction can be done efficiently without perturbing the guided mode too much. In order to avoid gap plasmons to build up in the slits 69 , a large enough $a_{\mathrm{G}}$ value is needed. We choose $a_{\mathrm{G}}=\frac{1}{3} d_{\mathrm{G}}$ here. Starting from $h_{\mathrm{G}}=2 \mathrm{~nm}$ we have increased the grating depth until an efficient coupling to the SP mode was found - we relied on a pure RCWA method 65, 66] to adjust this parameter.

It is easy to associate a diffraction order $m$ for most of the resonances supported by the grating described above (see Fig. 5). However, starting from almost zero and progressively increasing the height $h_{\mathrm{G}}$, the resonance that we first attributed to be of order $m=1$ exhibits a splitting

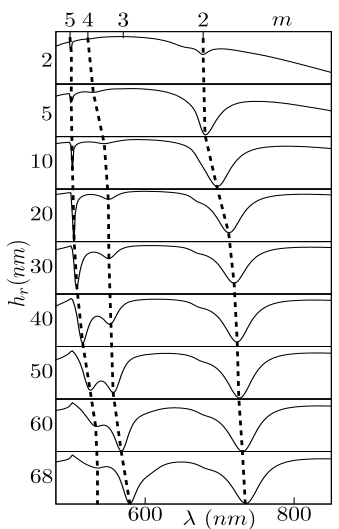

(a)

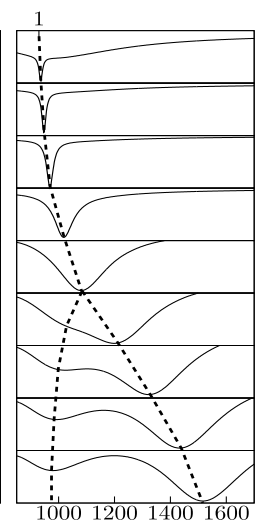

(b)

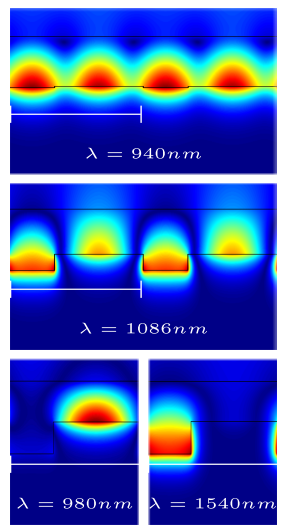

(c)
FIG. 5. (a): Reflectivity of the grating illuminated in normal incidence for different values of $h_{\mathrm{G}}$ computed using a local RCWA for $\lambda \in[480,850] \mathrm{nm}$. (b): Reflectivity of the grating for different values of $h_{\mathrm{G}}$ for $\lambda \in[850,1700] \mathrm{nm}$. Each vertical coordinate system has been chosen to maximize visibility. The horizontal one is kept constant from top to bottom. (c): Magnetic field amplitudes illustrating the splitting of diffraction order 1 into two sub-orders. The corresponding $\left(h_{\mathrm{G}}, \lambda\right)$ couples (in $\mathrm{nm}$ ) are from top to bottom : $(2,940),(30,1086)$, $(68,980)$ on the left and $(68,1540)$ on the right.

(see Fig. 5).

The field maps on Fig. 5 clearly show that one of the resonances corresponds to a cavity-like resonance, which is entirely located in the grooves of the grating, i.e it is reflected back and forth horizontally. The other resonances are cavity resonances of SPs that propagate on top of the grating and are reflected by the edges of the metal. We label those resonances with $1 b$ (where ' $b$ ' stands for 'bottom') and 1t (where 't' stands for 'top'), respectively. Since the thickness of the dielectric is different for both kinds of plasmons, their wavevector cannot be the same, therefore the splitting. We finally chose to take $h_{\mathrm{G}}=68$ nm.

Were the depth of the grating much larger, the $1 \mathrm{t}$ mode would enter a spoof SP regime 70 76 where the grating could be considered as an effective medium. In the present case, the conditions to be in this regime 73 are however not fulfilled, as the pitch is roughly one third of the wavelength at most - for the $1 \mathrm{t}$ mode. In addition, the grating is too shallow to allow for spoof SPs to be excited, making $1 \mathrm{~b}$ and $1 \mathrm{t}$ modes more similar to the hybrid SPs evoked in the literature [70. As the following will show, the 1t mode can be considered as insensitive to nonlocality, suggesting that spoof SPs may be equally insensitive. 


\section{B. Influence of nonlocality on the grating reflectivity}

Now that the grating has been designed and its physics is well understood, we use a software taking spatial dispersion into account in order to assess its influence on the reflectivity of the grating. This is necessary, since analytical solutions do not exist anymore and we have to rely on a numerical algorithm. Here, we use a DGTD method for the simulations of the grating. The result is shown in Fig. 6 for wavelengths ranging from $\lambda=500$ $\mathrm{nm}$ to $\lambda=2000 \mathrm{~nm}$. The small discrepancy between local DGTD and RCWA is due to a known problem of the latter in very peculiar conditions 77] and occurs only far off the resonances. We can clearly identify 5 dips due to SP excitation corresponding to 4 diffraction orders, the first order being split as discussed above. The results show a considerable influence of the spatial dispersion, which is significantly stronger than what has been predicted theoretically. This can be attributed to the grating itself and to the shift of the resonances towards large wavelengths (and larger wavevectors for the SP). The resonances linked to the diffraction orders $m=4$ and $m=2$ (see inset on Fig. 6) experience blueshifts of respectively $1.8 \mathrm{~nm}$ and $6.5 \mathrm{~nm}$ compared to the local prediction. Especially for $m=2$ this is clearly higher than the expected shift of $3 \mathrm{~nm}$ (see Fig. 4(b)). For $m=4$ the shift is slightly smaller than expected. Finally, we can identify two wavelength bands of interest. The first one for $\lambda=[550,800] \mathrm{nm}$ (see inset) shows the highest difference between local and nonlocal simulations. It comprises two blueshifted diffraction orders, and the whole response between the two is also clearly affected by nonlocality - making this regime a good choice to estimate the parameter $\beta$. The second region of interest is the rightmost part of Fig. 6 for $\lambda=[800,2000]$ $n m$. It comprises the two suborders $1 t$ and $1 b$, which are less sensitive to nonlocality. For this reason, we believe that this region is not useful to probe nonlocality, but well-suited for a geometrical parameter characterization. We have to keep in mind that any estimation of $\beta$ relies merely on a comparison between material models. Such comparisons are very sensitive to the geometrical parameters. Since we are trying to measure discrepancies of the order of $1 \%$ of the wavelength, we must ensure that nonlocality will not be concealed by uncertainties on local parameters.

\section{TELEMETRY AND PARAMETER ESTIMATION}

In the previous section we have theoretically predicted that the metallic grating that we have designed is sensitive to nonlocality. The goal of the present section is to present the challenges which would have to be faced by experimentalists and to propose a methodology relying on the solution of inverse problems and Uncertainty
Quantification (UQ), which could be used to assess realistically the presence of nonlocality and to estimate the crucial $\beta$ parameter.

In order to take into account the fact that, whatever the geometry and the imperfections of the grating, the spectra will be noisy, we have generated an artificial but realistic reflectance spectrum. We use nonlocal simulations and then added a noise whose characteristics are similar to actual experimental spectra provided by experimentalists [78. The noise has been chosen with a correlation length of $0.001 \mathrm{~nm}$ and a maximum difference of 0.04 with the original, unperturbed spectrum. We have then tested whether the parameters of the grating and of the model could be retrieved despite this realistic level of noise.

\section{A. Post-fabrication telemetry}

\section{Grating parameters}

The grating parameters would have to be determined before any dielectrics is deposited. A natural way of determining these geometrical parameters would be to rely on a Scanning Electron Microscope (SEM) or an Atomic Force Microscope (AFM) which would both allow to directly measure the precise parameters for each of the grooves of the grating. However, for a structure without any dielectrics, the impact of nonlocality is negligible - which means that the geometrical parameters can be determined using telemetry, without any assumption on the non local parameter, i.e with a local model. The optical response of a grating actually depends more on average geometrical parameters [7], as the grooves may be all slightly different. Telemetry has the advantage of allowing the determination of these average parameters (especially the period of the grating), which will constitute our geometric model.

We generate an artificial measurement spectrum, that we denote $R_{\text {meas }}(\lambda)$. Furthermore, it is possible, for given $d_{\mathrm{G}}, a_{\mathrm{G}}$ and $h_{\mathrm{G}}$ to compute a theoretical spectrum $R(\lambda)$. We define the distance between the two spectra as

$$
\left\|R_{\text {meas }}(\lambda)-R(\lambda)\right\|_{L^{2}\left(\lambda_{1}, \lambda_{2}\right)}^{2}=\int_{\lambda_{1}}^{\lambda_{2}}\left(R_{\text {meas }}(\lambda)-R(\lambda)\right)^{2} \mathrm{~d} \lambda .
$$

The integration interval $\left[\lambda_{1}, \lambda_{2}\right]$ will be chosen differently in the following, depending on which parameters have to be retrieved. We performed multiple optimization runs with different algorithms (particle swarm, pattern search and a derivative-based optimizer combined with a kriging-based meta-model) in order to find the parameters $\left(d_{G}, a_{G}\right.$ and $\left.h_{G}\right)$ which would produce the minimum distance between the theoretical spectrum and the artificially generated one. We underline that we constrained the optimization to look for geometrical parameters in intervals which would be in accordance with the precision of the etching process. The fabrication tolerances of 


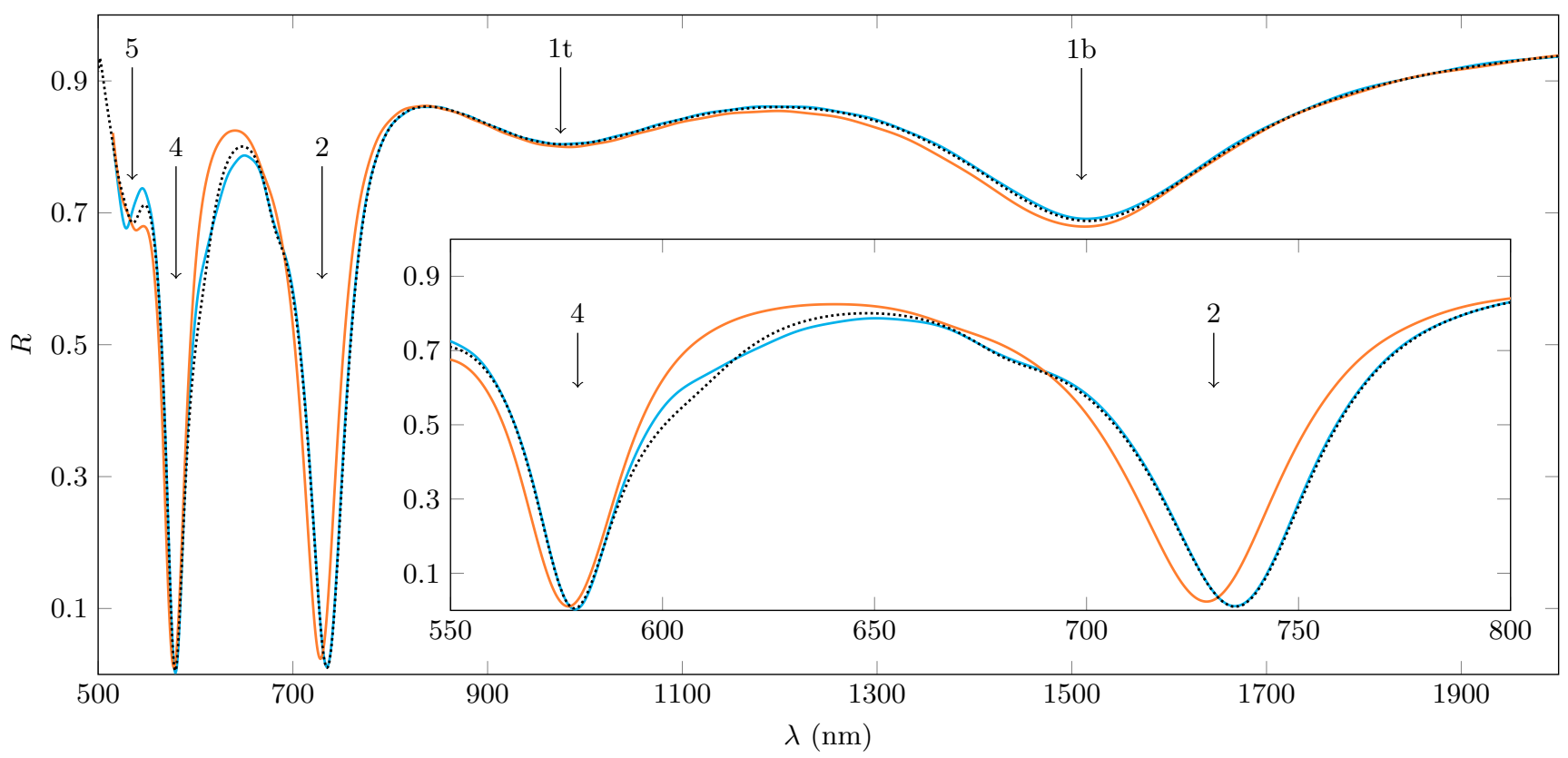

FIG. 6. Reflectivity $R$ of the whole structure as a function of the wavelength $\lambda$. The orange line corresponds to the nonlocal DGTD result, the blue line to the local DGTD result and the black, dotted line to the local RCWA result. The annotations 1t, 1b, 2, 4 and 5 refer to the excitation of the SP with respect to the diffraction order $m$ of 12 . The inset plot zooms into the wavelength range $550-800 \mathrm{~nm}$, where the impact of nonlocality is the most prominent.

state-of-the-art nano-processing still lead to a priori tolerances of about $\pm 5 \mathrm{~nm}$ for the etching process [80] and about $\pm 11 \%$ [81] for the dielectric deposition.

Considering spectra between $400 \mathrm{~nm}$ and $1200 \mathrm{~nm}$ to retrieve the parameters, we have found that the derivative-based optimization method performed best, followed by pattern search and particle swarm, the latter seemingly being less appropriate for this type of optimization problem. The geometrical parameters could be retrieved with an excellent accuracy despite the noise (see Fig 7).

\section{Dielectric thickness}

The dielectric thickness has to be determined by telemetry. While the previous step can be performed with purely local simulations, here nonlocality clearly plays a role. This time, our artificially measured data are generated using the nonlocal spectrum corresponding to the right part of Fig. 6 i.e $\left[\lambda_{1}, \lambda_{2}\right]=[800,2000]$ $\mathrm{nm}$. Since the resonances $1 \mathrm{t}$ and $1 \mathrm{~b}$ are almost insensitive to nonlocality, trying to retrieve $h_{\mathrm{D}}$ by minimizing the distance between the measured non local spectrum and a local in this wavelength range makes sense and actually yields results that are very close to the real value (the retrieved value was $h_{\mathrm{D}}=84.8 \mathrm{~nm}$, with the real value being $h_{\mathrm{D} \text {,init }}=85 \mathrm{~nm}$ ).

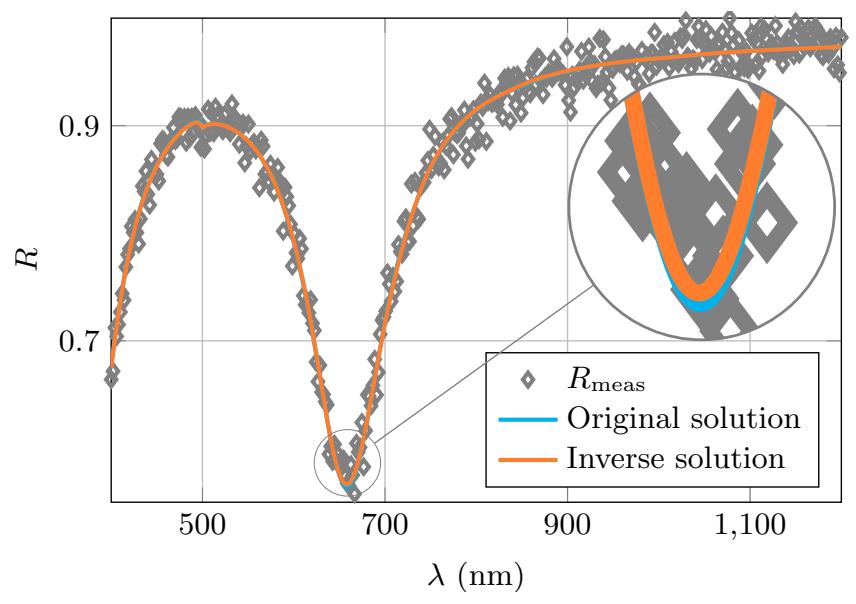

FIG. 7. Reflectivity spectrum. An artificial white noise has been added to the original spectrum (in blue) obtained for a grating illuminated in normal incidence without any dielectric layer and with $h_{\mathrm{G}}=68.0 \mathrm{~nm}, d_{\mathrm{G}}=500.00 \mathrm{~nm}$ and $a_{\mathrm{G}}=$ $166.7 \mathrm{~nm}$. In orange: The result of the optimization. This spectrum corresponds to $h_{\mathrm{G}}=68.1 \mathrm{~nm}, d_{\mathrm{G}}=499.2 \mathrm{~nm}$ and $a_{\mathrm{G}}=165.4 \mathrm{~nm}$. The constraint intervals are chosen to be $h_{\mathrm{G}} \in[62,73] \mathrm{nm}, d_{\mathrm{G}} \in[495,505] \mathrm{nm}$ and $a_{\mathrm{G}} \in[161,171] \mathrm{nm}$.

\section{B. Geometric uncertainty vs. nonlocality}

The geometric telemetry has led to the fabricated geometries in Table I] where, for each parameter $z$ with initial values $z_{\text {init }}$ used to create $R_{\text {meas }}, \bar{z}=z_{\text {opt }}$ is the mean value and $\delta_{z}=\left|\bar{z}-z_{\text {init }}\right|$ the maximum deviation. 
Given the uncertainties due to the retrieval process (with other means of measuring the geometric parameters these uncertainties would likely be of the same magnitude), we need to be sure that we will be able to distinguish the impact of nonlocality from an unavoidable small error in the determination of the geometric parameters. We use Uncertainty Quantification (UQ) to provide us with answers and, in order to estimate the impact of nonlocality we use, again, a $\beta$ value from the literature 39.

We recast the geometrical parameters $h_{\mathrm{G}}, d_{\mathrm{G}}, a_{\mathrm{G}}$ and $h_{\mathrm{D}}$ as random variables (RVs) following uniform distributions $\mathcal{U}\left[\bar{z}-\delta_{z}, \bar{z}+\delta_{z}\right]$ (seeI). We then perform an UQ 82 study, in order to estimate the expectation value and variance of the reflectivity in two resonance regions corresponding to the diffraction orders 2 and 4 . In particular, we consider 25 wavelengths in the range $[550,600] \mathrm{nm}$ $(m=4)$ and 50 wavelengths in the range $[700,800] \mathrm{nm}$ $(m=2)$.

Since the underlying computational model is a complex one, we rely on black-box UQ methods, i.e. the model and its numerical solvers are used without any modifications. In the context of the present work, we employ a spectral method [83, 84, in particular the stochastic collocation method 85 87, taking advantage of a number of factors. First of all, we deal with a small number of RVs, therefore the costs of the method remain affordable. Moreover, numerical tests indicate that the dependence of the reflectivity upon the RVs is smooth, which is a prerequisite for fast convergence. Finally, we assume that the RVs are mutually independent, which greatly simplifies the method's implementation.

The first step is to choose $M$ different set of values for the geometrical parameters $\mathbf{z}=\left(h_{\mathrm{G}}, d_{\mathrm{G}}, a_{\mathrm{G}}, h_{\mathrm{D}}\right)$, called the collocation points. For each wavelength mentioned above, the reflectance $R(\lambda)$ can be considered as a function $f(z)$ which is approximated by

$$
f(\mathbf{z}) \approx \sum_{m=1}^{M} f\left(\mathbf{z}^{(m)}\right) \Psi_{m}(\mathbf{z}),
$$

where $\mathbf{z}^{(m)}$ are realizations of the random vector (the collocation points) and $\Psi_{m}$ are multivariate Lagrange polynomials. The collocation points are based on univariate Clenshaw-Curtis quadrature nodes and are produced by Smolyak sparse grid rules [88, 89. The multivariate polynomials are formatted as products of univariate Lagrange polynomials, defined by the corresponding univariate Clenshaw-Curtis nodes.

TABLE I. Uncertain parameters extracted from telemetry

\begin{tabular}{|c|c|c|c|}
\hline Parameter & $\bar{z}$ & $\delta_{z}$ & Units \\
\hline$\overline{\overline{h_{\mathrm{G}}}}$ & 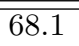 & $\overline{0.1}$ & $\overline{(\mathrm{nm})}$ \\
\hline$d_{\mathrm{G}}$ & 499.2 & 1.0 & $(\mathrm{~nm})$ \\
\hline$a_{\mathrm{G}}$ & 165.4 & 1.5 & $(\mathrm{~nm})$ \\
\hline$h_{\mathrm{D}}$ & 84.8 & 0.3 & $(\mathrm{~nm})$ \\
\hline
\end{tabular}

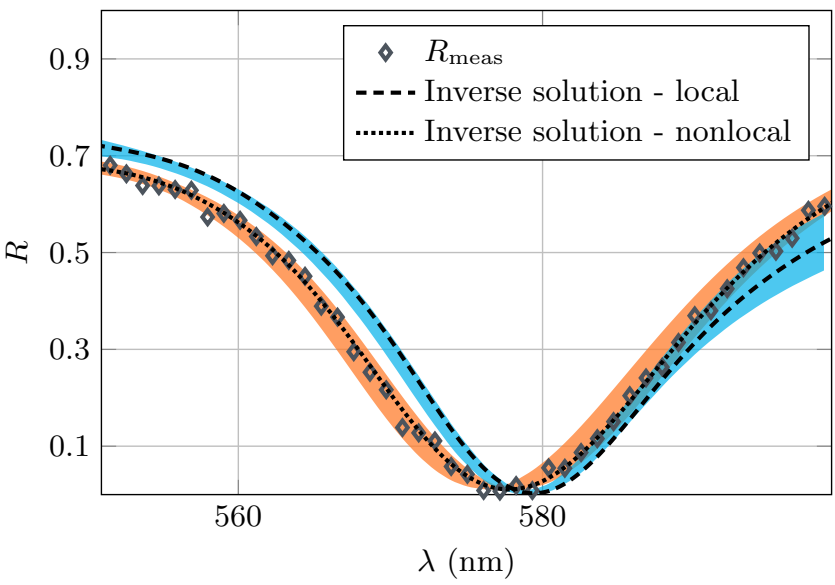

FIG. 8. Comparison of the local and nonlocal reflectivity for diffraction order $m=4$. The positions of the local resonances are $579 \mathrm{~nm}$ and $577 \mathrm{~nm}$ for the nonlocal one. This leads to a blueshift of almost $2 \mathrm{~nm}$. In blue: the $\mathbb{E}[R] \pm 2 \sigma$ area, being an output of the UQ analysis based on a stochastic collocation method. In orange: the min-max intervals of the reflectivity for all interval bound combinations of the geometrical parameters given in Table 1

The reflectivity's expectation value $\mathbb{E}[R]$ and variance $\mathbb{V}[R]$ can now be estimated by post-processing the approximation terms of 14 . Starting with the definitions

$$
\begin{aligned}
& \mathbb{E}[R]=\int_{\Gamma} f(\mathbf{z}) \varrho(\mathbf{z}) \mathrm{d} \mathbf{z}, \\
& \mathbb{V}[R]=\mathbb{E}\left[(R-\mathbb{E}[R])^{2}\right]=\mathbb{E}\left[R^{2}\right]-(\mathbb{E}[R])^{2},
\end{aligned}
$$

where $\varrho(\mathbf{z})$ is the joint probability density function, we approximate the corresponding integrals with the multidimensional Gauss quadrature formulas

$$
\begin{aligned}
& \mathbb{E}[R] \approx \sum_{m=1}^{M} w_{m} f\left(\mathbf{z}^{(m)}\right), \\
& \mathbb{V}[R] \approx \sum_{m=1}^{M} w_{m}\left(f\left(\mathbf{z}^{(m)}\right)-\mathbb{E}[R]\right)^{2},
\end{aligned}
$$

where $w_{m}$ denote the corresponding quadrature weights. We use the UQ study results in order to estimate $\pm 2 \sigma$ intervals around the optimized local reflectivity curve, where $\sigma=\sqrt{\mathbb{V}[R]}$ refers to the standard deviation. The results corresponding to each resonance area are presented in Fig. 8 and Fig. 9 (in blue), respectively. Since the nonlocal DGTD simulations are too expensive for an UQ of the same kind as we have performed for the local model, i.e. the evaluation of (17) and (18), we rely on a min-max study. Here, min-max represents solver calls for all interval bound combinations (in orange) as depicted in the corresponding figures.

According to Fig. 8, a clear measurement of the resonance $m=4$ is almost impossible due to the small difference between the local and nonlocal curves. Neverthe- 


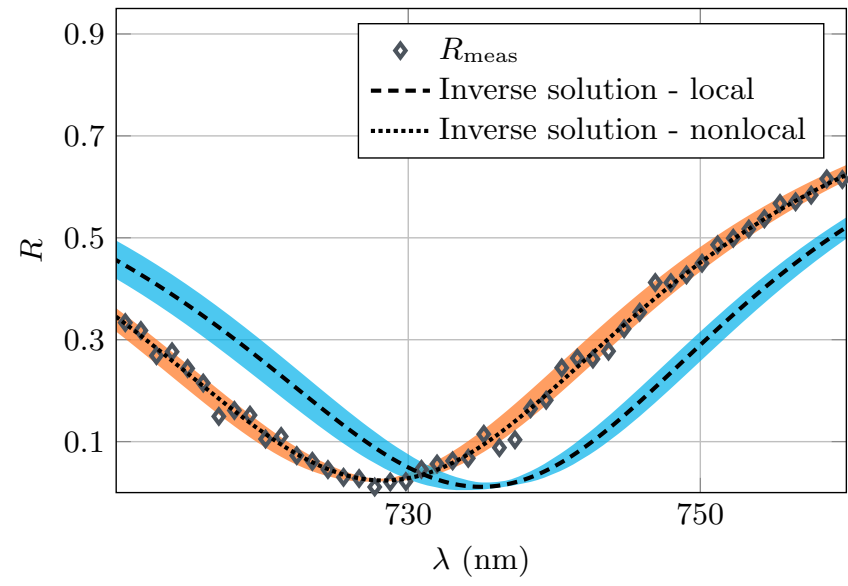

FIG. 9. Same as Fig. 8 for order of diffraction $m=2$. The positions of the local resonances is $735 \mathrm{~nm}$ and the nonlocal ones at $728 \mathrm{~nm}$. This leads to a blueshift of almost $7 \mathrm{~nm}$.

less, the second resonance, i.e. $m=2$ (see Fig. 9 ) is significantly more sensitive to nonlocality and stays distinguishable. For the sake of robustness, we have also performed simulations (data not shown) with the lowest theoretically acceptable value for $\beta=0.85 \times 10^{6} \mathrm{~m} . \mathrm{s}^{-1}$ [90], which still guarantees a blueshift of $5 \mathrm{~nm}$ for the second resonance, i.e. stronger than the geometric uncertainty.

\section{Model calibration}

Knowing the geometry and estimating the impact of nonlocality to be greater than geometric uncertainties, we now want to extract the nonlocal material parameter $\beta$. We underline that different theoretical expressions can be found in the literature for this constant [43, 49, 90, so that the theoretically acceptable values for $\beta$ may lie between $\beta_{\min } \approx 0.85 \times 10^{6} \mathrm{~m} . \mathrm{s}^{-1}\left[90\right.$ and $\beta_{\max } \approx 1.4 \times 10^{6}$ $\mathrm{m} . \mathrm{s}^{-1}$. 43. However, the experimental results available so far 39, 40] point consistently towards a value close to the upper estimation of $\beta=1.35 \times 10^{6} \mathrm{~m} . \mathrm{s}^{-1}$. This underlines how important the determination of $\beta$ can be and explains why we have considered this value so far.

In order to estimate how precise our estimation of $\beta$ could be with the grating setup, we proceed in the same fashion as for the geometric telemetry but we use a wavelength range of $[550,800] \mathrm{nm}$. Using DIOGENeS and DGTD [38, we find the $\beta$ value which minimizes the distance between $R$ and $R_{\text {meas }}$. The geometric size of the structure, in combination with the very small effective wavelengths and the short interaction range of nonlocal effects, which is in the range of several nm at the metallodielectric interface, result in a computationally expensive procedure. The solution of the inverse problem can be significantly accelerated by meta-model-based optimization algorithms. We have used the kriging (Gaussian process) meta-model in combination with a derivative-based optimization implementation of the FAMOSA 91 optimization toolbox. We find $\beta_{\text {inverse }}=1.385 \times 10^{6} \mathrm{~m} . \mathrm{s}^{-1}$, which is reasonably close to the $\beta_{\text {init }}=1.35 \times 10^{6} \mathrm{~m} . \mathrm{s}^{-1}$ (the value used to generate $R_{\text {meas }}$ ), indicating that the value of $\beta$ can be retrieved with an error smaller than $10 \%$.

\section{CONCLUSION}

We have first shown that, in the framework of the hydrodynamic model, SPs can be sensitive enough to spatial dispersion - provided that the dielectric considered has a sufficiently high permittivity, like $\mathrm{TiO}_{2}$. Such a conclusion is in contrast with previous works that suggested that the impact of spatial dispersion could be too difficult to measure optically - which is only true for an interface between metal and air.

Since there is a link between such guided modes and the localized resonances of metallic nanoparticles, this lead us to expect an impact of nonlocality on essentially any metallic nanoparticle with a radius much larger than $20 \mathrm{~nm}$, for which the geometry is more likely to be well controlled, buried in a high index medium. Using Mie theory, we estimate the blueshift brought by nonlocality to be at least of $5 \mathrm{~nm}$ in wavelength in $\mathrm{TiO}_{2}$, an effect that could potentially be observed experimentally.

Since high wavevector guided modes cannot be excited using prism couplers, we have then studied how the SPs can be excited using a grating coupler. We have shown, using state of the art numerical tools, that such a structure would allow the observation of spatial dispersion by means of blueshifted resonances up to almost $7 \mathrm{~nm}$ (around 1\% of the wavelength). Using uncertainty quantification and inverse problem solving, we have identified which resonance precisely could be used to estimate the main parameter of the hydrodynamic model and shown how such an estimation could be made. We underline that such a procedure could well be applied to nanoparticles as well.

As already evoked in several earlier works [59, 92, 93. and clearly demonstrated in the present work, any plasmonic structure surrounded by a high refractive index medium like $\mathrm{TiO}_{2}$ will only be accurately described if spatial dispersion is taken into account.

We believe that, by proposing a structure with realistic parameters and a procedure to carefully estimate the impact of spatial dispersion, this work will pave the way for future experiments that shall give reliable answers to the community on the limits of Drude's model for plasmonics and its potential replacements. 
[1] P. Drude, Annalen der Physik 306, 566 (1900).

[2] J. C. Maxwell, A treatise on electricity and magnetism, Vol. 1 (Clarendon press, 1881).

[3] A. Moreau, C. Ciracì, J. J. Mock, R. T. Hill, Q. Wang, B. J. Wiley, A. Chilkoti, and D. R. Smith, Nature 492, 86 (2012).

[4] X. Chen, C. Ciracì, D. R. Smith, and S.-H. Oh, Nano letters 15, 107 (2014).

[5] G. M. Akselrod, C. Argyropoulos, T. B. Hoang, C. Ciracì, C. Fang, J. Huang, D. R. Smith, and M. H. Mikkelsen, Nature Photonics 8, 835 (2014).

[6] A. W. Powell, D. M. Coles, R. A. Taylor, A. A. Watt, H. E. Assender, and J. M. Smith, Advanced Optical Materials 4, 634 (2016).

[7] M. Ayata, Y. Fedoryshyn, W. Heni, B. Baeuerle, A. Josten, M. Zahner, U. Koch, Y. Salamin, C. Hoessbacher, C. Haffner, et al., Science 358, 630 (2017).

[8] R. Fuchs and K. L. Kliewer, Phys. Rev. B 3, 2270 (1971)

[9] P. J. Feibelman, Physical Review B 12, 1319 (1975).

[10] M. Rocca, Surface science reports 22, 1 (1995).

[11] M. Rocca, L. Yibing, F. BuatierdeMongeot, and U. Valbusa, Physical Review B 52, 14947 (1995).

[12] S. J. Park and R. E. Palmer, Physical review letters 102, 216805 (2009).

[13] A. Liebsch, Physical Review B 48, 11317 (1993).

[14] A. Liebsch, Physical review letters 71, 145 (1993).

[15] P. J. Feibelman, Phys. Rev. Lett. 72, 788 (1994)

[16] A. Liebsch, Phys. Rev. Lett. 72, 789 (1994)

[17] A. D. Boardman, Electromagnetic surface modes (Wiley, 1982).

[18] F. Frostmann and R. R. Gerhardts, Metal optics near the plasma frequency, Vol. 109 (Springer-Verlag, 1986).

[19] R. Chang, H.-P. Chiang, P. Leung, and W. Tse, Optics Communications 225, 353 (2003)

[20] J. A. Scholl, A. L. Koh, and J. A. Dionne, Nature 483 , 421 (2012).

[21] S. Raza, S. I. Bozhevolnyi, M. Wubs, and N. A. Mortensen, Journal of Physics: Condensed Matter 27, 183204 (2015).

[22] R. Esteban, A. G. Borisov, P. Nordlander, and J. Aizpurua, Nature communications 3, 825 (2012).

[23] C. Ciracì, J. B. Pendry, and D. R. Smith, ChemPhysChem 14, 1109 (2013).

[24] A. Moreau, C. Ciracì, and D. R. Smith, Physical Review B 87, 045401 (2013).

[25] S. Raza, T. Christensen, M. Wubs, S. Bozhevolnyi, and N. Mortensen, Physical Review B 88, 115401 (2013).

[26] C. David, N. A. Mortensen, and J. Christensen, Scientific reports 3, 2526 (2013).

[27] A. Wiener, A. I. Fernández-Domínguez, J. Pendry, A. P. Horsfield, and S. A. Maier, Optics Express 21, 27509 (2013).

[28] G. Toscano, S. Raza, W. Yan, C. Jeppesen, S. Xiao, M. Wubs, A.-P. Jauho, S. I. Bozhevolnyi, and N. A. Mortensen, Nanophotonics 2, 161 (2013).

[29] J. B. Lassiter, X. Chen, X. Liu, C. Ciracì, T. B. Hoang, S. Larouche, S.-H. Oh, M. H. Mikkelsen, and D. R. Smith, Acs Photonics 1, 1212 (2014).

[30] C. Haffner, W. Heni, Y. Fedoryshyn, J. Niegemann, A. Melikyan, D. L. Elder, B. Baeuerle, Y. Salamin, A. Josten, U. Koch, et al., Nature Photonics 9, 525
(2015).

[31] M. P. Nielsen, X. Shi, P. Dichtl, S. A. Maier, and R. F. Oulton, Science 358, 1179 (2017).

[32] H. Haberland, Nature 494, E1 (2013).

[33] G. Toscano, J. Straubel, A. Kwiatkowski, C. Rockstuhl, F. Evers, H. Xu, N. A. Mortensen, and M. Wubs, Nature communications 6, 7132 (2015).

[34] C. Ciracì and F. Della Sala, Physical Review B 93, 205405 (2016).

[35] G. Toscano, S. Raza, A.-P. Jauho, N. A. Mortensen, and M. Wubs, Optics express 20, 4176 (2012).

[36] J. Benedicto, R. Pollès, C. Ciracì, E. Centeno, D. R. Smith, and A. Moreau, JOSA A 32, 1581 (2015).

[37] N. Schmitt, C. Scheid, S. Lanteri, A. Moreau, and J. Viquerat, Journal of Computational Physics 316, 396 (2016).

[38] N. Schmitt, C. Scheid, J. Viquerat, and S. Lanteri, Journal of Computational Physics 373, 210 (2018).

[39] C. Ciracì, R. T. Hill, J. J. Mock, Y. Urzhumov, A. I. Fernández-Domínguez, S. A. Maier, J. B. Pendry, A. Chilkoti, and D. R. Smith, Science 337, 1072 (2012).

[40] C. Ciracì, X. Chen, J. J. Mock, F. McGuire, X. Liu, S.H. Oh, and D. R. Smith, Applied Physics Letters 104, 023109 (2014)

[41] S. Raza, N. Stenger, S. Kadkhodazadeh, S. V. Fischer, N. Kostesha, A.-P. Jauho, A. Burrows, M. Wubs, and N. A. Mortensen, Nanophotonics 2, 131 (2013).

[42] A. D. Rakic, A. B. Djurišic, J. M. Elazar, and M. L. Majewski, Applied Optics 37, 5271 (1998).

[43] S. Raza, G. Toscano, A. P. Jauho, M. Wubs, and N. A. Mortensen, Physical Review B - Condensed Matter and Materials Physics 84 (2011), 10.1103/PhysRevB.84.121412, arXiv:arXiv:1106.2175v2.

[44] P.-O. Chapuis, S. Volz, C. Henkel, K. Joulain, and J.-J. Greffet, Physical Review B 77, 035431 (2008).

[45] R. Ruppin, Journal of Physics: Condensed Matter 17, 1803 (2005).

[46] R. Ruppin and K. Kempa, Physical Review B 72, 153105 (2005).

[47] A. Wiener, A. I. Fernndez-Domnguez, A. P. Horsfield, J. B. Pendry, and S. A. Maier, Nano Letters 12, 3308 (2012), http://pubs.acs.org/doi/pdf/10.1021/nl301478n.

[48] C. Ciracì, Y. Urzhumov, and D. R. Smith, JOSA B 30, 2731 (2013).

[49] N. Crouseilles, P. A. Hervieux, and G. Manfredi, Phys. Rev. B 78, 155412 (2008).

[50] A. A. Maradudin and D. L. Mills, Phys. Rev. B 7, 2787 (1973)

[51] S. Lanteri, C. Scheid, and J. Viquerat, SIAM Journal on Scientific Computing 39, A831 (2017)

[52] DIOGENeS, https://diogenes.inria.fr/.

[53] R. W. Wood, Proceedings of the Physical Society of London 18, 269 (1902)

[54] U. Kreibig and M. Vollmer, Optical properties of metal clusters, Vol. 25 (Springer Science \& Business Media, 2013).

[55] T. Klar, M. Perner, S. Grosse, G. Von Plessen, W. Spirkl, and J. Feldmann, Physical Review Letters 80, 4249 (1998).

[56] M. Scalora, M. A. Vincenti, D. de Ceglia, V. Roppo, M. Centini, N. Akozbek, and M. J. Bloemer, Phys. Rev. 
A 82, 043828 (2010).

[57] J. A. Scholl, A. García-Etxarri, A. L. Koh, and J. A. Dionne, Nano letters 13, 564 (2013).

[58] T. V. Teperik, P. Nordlander, J. Aizpurua, and A. G. Borisov, Physical review letters 110, 263901 (2013).

[59] S. Raza, S. Kadkhodazadeh, T. Christensen, M. Di Vece, M. Wubs, N. A. Mortensen, and N. Stenger, Nature Communications 6, 8788 (2015).

[60] N. A. Mortensen, S. Raza, M. Wubs, T. Søndergaard, and S. I. Bozhevolnyi, Nature communications 5, 3809 (2014).

[61] R. Ruppin, Optics Communications 190, 205 (2001)

[62] T. Siefke, S. Kroker, K. Pfeiffer, O. Puffky, K. Dietrich, D. Franta, I. Ohldal, A. Szeghalmi, E.-B. Kley, and A. Tnnermann, Advanced Optical Materials 4, 1780 (2016)

[63] D. Maystre, Scholarpedia 7, 11403 (2012).

[64] J. Defrance, C. Lemaître, R. Ajib, J. Benedicto, É. Mallet, R. Pollès, J.-P. Plumey, M. Mihailovic, E. Centeno, C. Ciracì, D. Smith, and A. Moreau, Journal of Open Research Software 4 (2016).

[65] G. Granet and B. Guizal, J. Opt. Soc. Am. A 13, 1019 (1996).

[66] P. Lalanne and G. M. Morris, J. Opt. Soc. Am. A 13, 779 (1996).

[67] J. R. Maack, N. A. Mortensen, and M. Wubs, EPL (Europhysics Letters) 119, 17003 (2017).

[68] D. Gall, Journal of Applied Physics 119, 085101 (2016), https://doi.org/10.1063/1.4942216

[69] M. Dechaux, P.-H. Tichit, C. Ciracì, J. Benedicto, R. Pollès, E. Centeno, D. R. Smith, and A. Moreau, Physical Review B 93, 045413 (2016).

[70] J. Pendry, L. Martin-Moreno, and F. Garcia-Vidal, Science 305, 847 (2004).

[71] A. B. Khanikaev, S. H. Mousavi, G. Shvets, and Y. S. Kivshar, Physical review letters 105, 126804 (2010).

[72] A. Rusina, M. Durach, and M. I. Stockman, Applied Physics A 100, 375 (2010).

[73] M. A. Kats, D. Woolf, R. Blanchard, N. Yu, and F. Capasso, Optics express 19, 14860 (2011).

[74] A. Pors, E. Moreno, L. Martin-Moreno, J. B. Pendry, and F. J. Garcia-Vidal, Physical review letters 108, 223905 (2012).

[75] B. Ng, J. Wu, S. M. Hanham, A. I. FernándezDomínguez, N. Klein, Y. F. Liew, M. B. Breese, M. Hong, and S. A. Maier, Advanced Optical Materials 1, 543 (2013).

[76] X. Shen and T. J. Cui, Laser \& Photonics Reviews 8, 137 (2014).

[77] L. Li and G. Granet, J. Opt. Soc. Am. A 28, 738 (2011).

[78] O. Jamadi, F. Reveret, P. Disseix, F. Medard, J. Leymarie, A. Moreau, D. Solnyshkov, C. Deparis, M. Leroux, E. Cambril, et al., Light: Science \& Applications 7, 82 (2018).

[79] M. Kaliteevski, J. M. Martinez, D. Cassagne, and J. Albert, Physical Review B 66, 113101 (2002).

[80] E. Langereis, S. B. S. Heil, H. C. M. Knoops, W. Keuning, M. C. M. van de Sanden, and W. M. M. Kessels, Journal of Physics D: Applied Physics 42, 073001 (2009)

[81] Y. Chen, Microelectronic Engineering 135, 57 (2015)

[82] R. C. Smith, Uncertainty Quantification - Theory, Implementation, and Applications., Computational science and engineering (SIAM, 2014) pp. I-XVIII, 1-382.
[83] O. P. Le Maitre and O. M. Knio, Spectral Methods for Uncertainty Quantification: With Applications to Computational Fluid Dynamics, Scientific Computation (Springer Netherlands, 2010).

[84] D. Xiu, Numerical Methods for Stochastic Computations: A Spectral Method Approach (Princeton University Press, Princeton, NJ, USA, 2010).

[85] I. Babuska, F. Nobile, and R. Tempone, SIAM Review , 317 (2010).

[86] F. Nobile, R. Tempone, and C. G. Webster, SIAM J. Numerical Analysis 46, 2309 (2008).

[87] D. Xiu and J. S. Hesthaven, SIAM J. Scientific Computing 27, 1118 (2005).

[88] H.-J. Bungartz and M. Griebel, Acta Numerica 13, 147 (2004)

[89] S. A. Smolyak, Dokl. Acad. Nauk SSSR 4, 240 (1963).

[90] K. R. Hiremath, L. Zschiedrich, and F. Schmidt, Journal of Computational Physics 231, 5890 (2012), arXiv:1201.1527

[91] R. Duvigneau, "FAMOSA, http://wwwsop.inria.fr/members/Regis.Duvigneau/soft_famosa.html,".

[92] A. Pitelet, É. Mallet, E. Centeno, and A. Moreau, Phys. Rev. B 96, 041406 (2017).

[93] A. Pitelet, É. Mallet, R. Ajib, C. Lemaître, E. Centeno, and A. Moreau, Phys. Rev. B 98, 125418 (2018) 